\title{
Presencia de la rata invasora Rattus rattus (Rodentia: Muridae) en el Parque Nacional Sangay, Ecuador
}

Jorge Brito ${ }^{1 *}$ y Reed Ojala-Barbour ${ }^{2}$

Introduction: The black rat Rattus rattus is one of the most invasive and harmful mammals in the world. The species is usually commensal with humans and is distributed throughout the world except in polar regions. As far as is known, $R$. rattus has not previously been captured in pristine rainforests in Ecuador. A recent small mammal survey in the rainforest of Sangay National Park (SNP) revealed the presence of the species in this important ecosystem, which has been designated a Natural World Heritage Site by UNESCO.

Methodology: Sampling was conducted during six expeditions at four sites in SNP (La Libertad, Sardinayacu, Zuñac and Tinguichaca). The trapping effort in La Libertad and Zuñac was three days each, and in Tinguichaca and Sardinayacu six days each, in both the rainy season (February-May) and dry season (July-October). To capture small terrestrial mammals, 100 traps were used at each site (three nights per visit at two sites and six nights in the other two sites), with a total sampling effort of 1,800 traps per night.

Results: In April 2012 we captured one male Rattus rattus in Sardinayacu in a trap set $1.5 \mathrm{~m}$ above ground level.

Discussion and conclusion: It is difficult to accurately determine how the rat moved into this pristine forest, but possibly it traveled along a path used by tourists and rangers that originates in a community $17 \mathrm{Km}$ away which goes through a secondary forest, pasture and then into the SNP to Sardinayacu. It is possible that the invasive rat $R$. rattus, which is common in disturbed environments, may be moving into pristine environments (Wolf et al, 2005), which would affect native fauna.

Key words: Eastern Mountain System, invasive species, Rattus rattus, Sangay National Park, Subtropical rainforest.

\section{Resumen}

Registramos por primera vez la presencia de la rata invasora Rattus rattus en Sardinayacu, dentro del Parque Nacional Sangay, al sureste de Ecuador. Esta área protegida presenta bosque húmedo prístino (alejado de la presencia humana) en la Cordillera Oriental de Los Andes. La información presentada se basa en un ejemplar recolectado en la localidad de Sardinayacu, Parque Nacional Sangay y destaca su importancia porque no existen reportes científicos previos para esta especie en bosques húmedos prístinos. Discutimos porqué la presencia de Rattus rattus en este importante ecosistema, denominado por la UNESCO como Patrimonio Natural de la Humanidad, podría causar daños a la fauna nativa.

\footnotetext{
${ }^{1}$ Museo Ecuatoriano de Ciencias Naturales. División de Mastozoología. Calle Rumipamba 341 y Av. de los Shyris. Casilla: 17-07-8976. Quito, Ecuador. E-mail: jorgeyakuma@yahoo.es (JB)

${ }^{2}$ Instituto de Ciencias Biológicas, Escuela Politécnica Nacional. Ladrón de Guevara E11-253. Casilla: 17-01-2759. Quito, Ecuador. E-mail: reed.ojala.barbour@fulbrightmail.org (ROB)

"Corresponding author
} 
Palabras clave: Bosque húmedo, Cordillera Oriental, especie invasora, Parque Nacional Sangay, Rattus rattus.

Introducción

La introducción de especies fuera de su hábitat original se remonta a antiguas civilizaciones y siempre ha acompañado los procesos de colonización (Gutiérrez 2006). Las especies introducidas e invasoras se pueden encontrar a todo nivel, entre microorganismos, plantas terrestres y acuáticas, invertebrados, anfibios, aves, peces, mamíferos y reptiles (Feinstein 2004). A nivel mundial, el 90\% de las introducciones de vertebrados y plantas son intencionales, y el restante $10 \%$ accidentales (Towsend et al. 2004). En islas oceánicas la extinción de reptiles, aves y roedores endémicos ha sido atribuida al impacto de las ratas introducidas (Amori y Clout 2002).

La rata invasora Rattus rattus Linnaeus 1785, es originaria de Asia, pero en la actualidad se encuentra distribuida alrededor de todo el mundo como especie comensal del hombre (Nowak 1991; Musser y Carleton 2005), excepto en las regiones polares (Shiels et al. 2013). A Ecuador se presume que llegó entre los siglos XVI o XVII en los barcos de los conquistadores españoles (Tirira 2007). En el 2001 la Unión Internacional para la Conservación de la Naturaleza (IUCN por sus siglas en inglés) catalogó a $R$. rattus dentro de las 100 especies invasoras más dañinas a nivel mundial y subregionalmente, entre los países Andinos (Bolivia, Colombia, Ecuador, Perú, Venezuela), R. rattus se encuentra dentro de los 30 vertebrados exóticos invasores (Ojasti 2001).

En Ecuador la especie se distribuye en varias islas del Archipiélago de Galápagos (Dexter et al. 2004); a nivel continental, se encuentra en áreas urbanas y rurales. Hasta donde se conoce, esta especie no ha sido capturada en el bosque húmedo prístino; no obstante, se ha registrado en los bordes e interior de bosques secos (Tirira 2007). A pesar de contar con una amplia literatura sobre el efecto de $R$. rattus en la biota de las Islas Galápagos (e. g. Clark 1980, 1981, 1982; Dexter et al. 2004; Harris y MacDonald 2007); se conocen poco sus efectos en el Ecuador continental; tan solo existen registros de patógenos en poblaciones urbanas y rurales (e. g. Pinto et al. 2003, 2006).

El Parque Nacional Sangay (PNS) es considerado como ecosistema intangible, muy preciado para la conservación e investigación científica biológica (INEFAN 1998). Se encuentra ubicado al sureste de Ecuador, cuenta con una superficie de 502,105 ha, un intervalo altitudinal entre los 1,000 a 5,230 msnm y presenta 10 formaciones vegetales (INEFAN 1998). Debido a la importancia biológica del PNS, se realizaron varias expediciones en el año 2012 para estudiar la diversidad y aspectos ecológicos de los mamíferos pequeños terrestres. Los muestreos se realizaron durante seis expediciones en cuatro sitios (La Libertad, Sardinayacu, Zuñac y Tinguichaca). El esfuerzo de trampeo en La Libertad y Zuñac fue de tres días en cada uno, y en Sardinayacu y Tinguichaca fue de seis días en cada uno, tanto en época de lluvia (febrero-mayo) como en época seca (julio-octubre). El registro de la rata invasora $R$. rattus dentro de este importante ecosistema motivó la realización del presente estudio.

El registro de la especie proviene de Sardinayacu en el interior del PNS, cantón Morona, provincia de Morona Santiago $\left(-2.0666^{\circ} \mathrm{S},-78.2166^{\circ} \mathrm{W}\right.$, a 1,776 msnm), ubicado de 
ocho a diez horas de camino a pie (aprox. $17 \mathrm{~km}$ ) del poblado más cercano, Playas de San Luis. El área se caracteriza por presentar un bosque maduro dominado por árboles de romerillo Prumnopitys montana y palma real Dictyocaryum lamarckianum.

Para la captura de los pequeños mamíferos terrestres en el PNS se usaron 100 trampas Sherman cebadas con avena por noche en cada sitio (tres noches por visita en dos sitios y seis noches en otros dos sitios), y se ubicaron sobre el suelo y a $2 \mathrm{~m}$ del mismo (en troncos horizontales). El esfuerzo total de muestreo fue de 1,800 trampas/noche.

El 19 de abril de 2012 se capturó un ejemplar de $R$. rattus a $1.5 \mathrm{~m}$ sobre el nivel del suelo. Este ejemplar era un macho y presentaba testículos abdominales. Las características morfológicas del individuo colectado fueron las siguientes: largo total 385 mm; largo de la cola 200 mm; peso 124 g; pelaje largo y grueso, poco denso; dorso gris oscuro, con los pelos de guardia que sobresalen; región ventral gris blancuzco; vibrisas gruesas, largas y cortas, alcanzan los hombros (Fig. 1). Las principales medidas craneales fueron las siguientes: longitud cóndilo incisivo $38 \mathrm{~mm}$; longitud serie molar superior $6.6 \mathrm{~mm}$; anchura caja craneana $16.6 \mathrm{~mm}$; anchura bicigomática $19.4 \mathrm{~mm}$; anchura interorbitaria mínima $6.1 \mathrm{~mm}$; longitud del rostro $6.7 \mathrm{~mm}$.

Los rasgos craneales más sobresalientes del individuo fueron los siguientes: primer molar superior (M1) presenta unas hendiduras externas en la primera fila de cúspides, y la longitud del parietal medida a lo largo del borde temporal es menor que la distancia entre los bordes temporales; éstas características distinguen a esta especie de $R$. norvegicus (Hall y Kelson 1959). La piel y el esqueleto de este espécimen (MEPN-12195) se depositaron en la sección de mastozoología del Instituto de Ciencias Biológicas de la Escuela Politécnica Nacional, Quito, Ecuador. Fue registrado en simpatría con las siguientes especies nativas de roedores cricétidos: Chilomys instans, Oreoryzomys balneator, Rhipidomys sp., e Hylaeamys tatei, ésta última es endémica de Ecuador (Musser et al. 1998).

En los ecosistemas insulares a nivel mundial, $R$. rattus es considerado como el roedor invasor más perjudicial (Ruffino et al. 2009; Traveset et al. 2009; Banks y Hughes 2012). Varios autores han identificado a las ratas del género Rattus, como uno de los principales factores de riesgo para las poblaciones de aves acuáticas de islas del noroeste de Baja California y del Golfo de California en donde han sido introducidas (Mellink 1992; Velarde y Anderson 1994; McChesney y Tershy 1998). En América del Sur existen casos puntales sobre el efecto de roedores introducidos en especies nativas; por ejemplo, en Argentina documentan que Rattus es depredador del ratón topo Chelemys macronyx (Shepherd y Rebecca 2012); mientras que en Chile estas ratas son depredadoras de aves terrestres (Jaksic 1998), y además atacan a huevos y aves en graneros, sugiriendo que lo mismo podría suceder en ambientes naturales (Lobos et al. 2005).

Las ratas, tanto en ambientes insulares, como en regiones continentales, pueden llegar a ser competidoras importantes de varias especies de pequeños mamíferos; asimismo, han sido identificadas como portadoras de numerosas enfermedades y parásitos transmisibles a la fauna nativa e incluso al ser humano (Álvarez-Romero y Medellín 2005; Harris y MacDonald 2007; Shiels et al. 2013). Además, son los principales causantes del mayor número de disminuciones o extinciones de la biota endémica en regiones insulares (Towns et al. 2006). La erradicación de ratas en ambientes insulares es factible, en las que es más difícil mantener poblaciones viables (Bertram y Nagorsen 1995), mientras 
que en ecosistemas como los bosques húmedos continentales la erradicación sería muy difícil, puesto que las técnicas empleadas (e. g. cebos envenenados) podrían afectar a la fauna nativa.

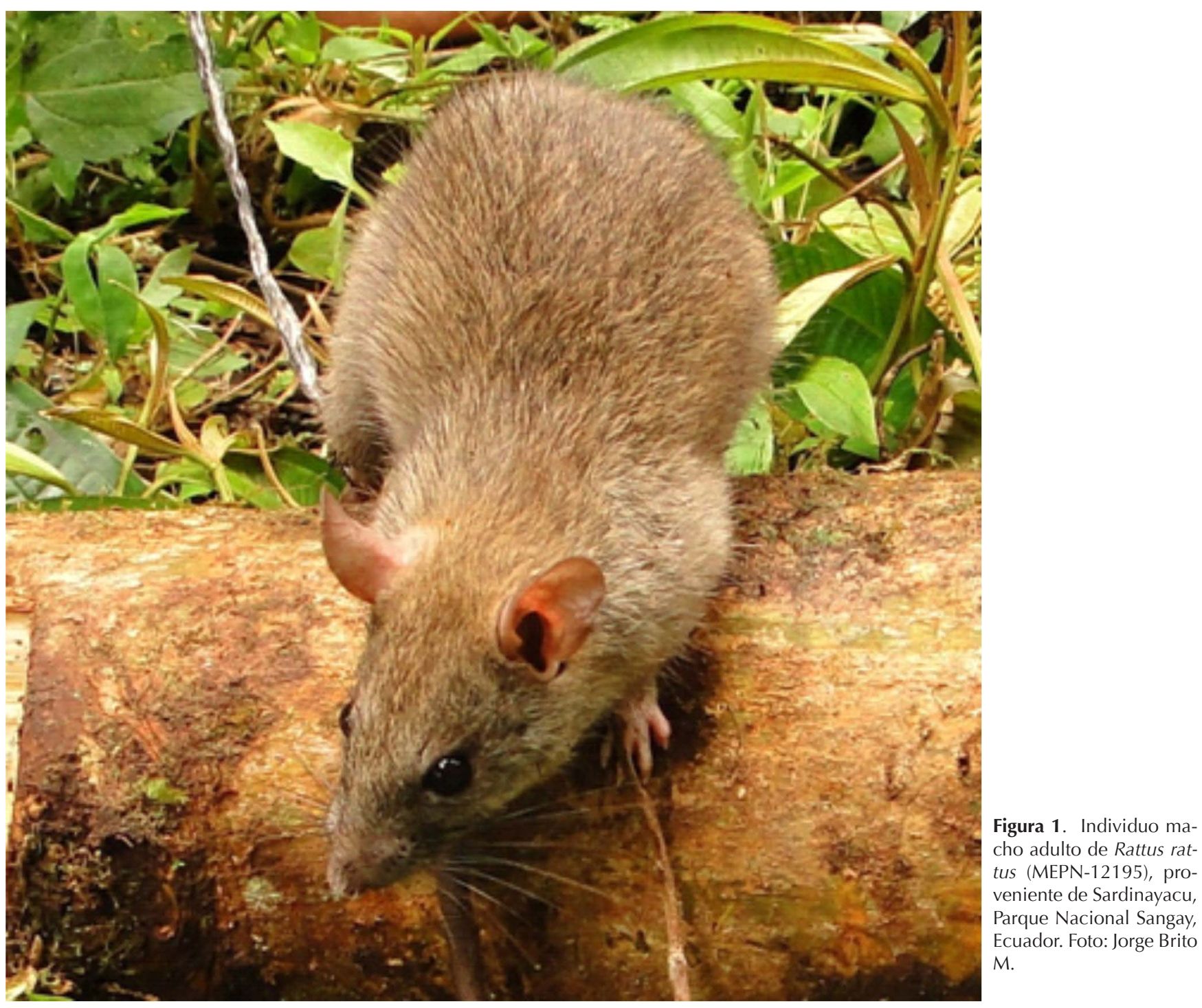

El único registro de $R$. rattus en el PNS reportado en este estudio, probablemente se deba a un reciente desplazamiento, puesto que durante varias expediciones realizadas para el estudio de los pequeños mamíferos en este importante ecosistema (Albuja et al. 1996; Lee et al. 2011), incluyendo áreas con intervención humana, no dieron a conocer a esta especie invasora dentro de sus reportes. En el estudio de Albuja et al. (1996), que incluyó un muestreo en el sendero Sardinayacu, a $500 \mathrm{~m}$ del borde hacia el interior del PNS (a 7.5 km de nuestro registro), no mencionó a $R$. rattus en su reporte. Resulta dificultoso determinar con exactitud el desplazamiento de la rata a los bosques prístinos de Sardinayacu. El sitio de registro se encuentra a $8 \mathrm{~km}$ de un borde de bosque, donde existe un pastizal con presencia de ganado vacuno. Posiblemente la rata se desplazó por un sendero usado por turistas y guardaparques, mismo que se origina a $17 \mathrm{~km}$ en un asentamiento humano, atraviesa un bosque secundario, un pastizal y luego se adentra en el PNS hasta Sardinayacu. Un caso similar reporta Lobos et al. (2005) en 
Chile, mencionando la presencia de la rata invasora $R$. rattus en dos parques nacionales en Chile, a $6.9 \mathrm{~km}$ de los asentamientos humanos, manifestando preferencia por los bosques húmedos y bosques de niebla.

Es necesario realizar estudios adicionales dentro del bosque prístino en el PNS, en áreas intervenidas y zonas pobladas aledañas, con la finalidad de determinar la presencia y comparar la densidad poblacional de $R$. rattus, usando diferentes tipos de técnicas como trampas de resorte y de caída. Una técnica adicional a emplearse podría ser la usada por Dexter et al. (2004), en las islas Galápagos, donde colocaron en las trampas Sherman fecas secas de gato doméstico (Felis silvestris cattus), con la finalidad de medir la evasión de ingreso a las trampas por $R$. rattus y la susceptibilidad a la depredación de los roedores endémicos por los gatos domésticos. Es posible que la rata invasora $R$. rattus, que es común en ambientes alterados, se estaría desplazando hacia ambientes prístinos (Lobos et al.v 2005), lo que podría afectar a la fauna nativa.

\section{Agradecimientos}

De manera especial a V. León, responsable del Parque Nacional Sangay, zona baja, por su valiosa colaboración con la logística para todas las expediciones. G. Pozo y M. Sharup (Churuwia) colaboraron en la fase de campo. Nuestro reconocimiento a W. R. Teska de la Universidad Luterana del Pacífico quien generosamente nos facilitó las trampas Sherman. C. M. Pinto, A. Arguero, P. Moreno, C. Lorenzo y dos revisores anónimos aportaron sus valiosos comentarios para el fortalecimiento de este estudio. La investigación fue realizada gracias a una beca concedida a ROB por la comisión Fulbright. Al Ministerio del Ambiente de Morona Santiago por otorgar el permiso de investigación Nº 04-2012-I-B-DPMS/MAE.

\section{Literatura citada}

Albuja, L., J. Palacios., M. Cueva, y G. Paredes. 1996. Diagnóstico faunístico para la actualización del plan de manejo del Parque Nacional Sangay. INEFAN, Proyecto GEF. Quito, Ecuador.

Átvarez-Romero, J., y R. A. Medeluín. 2005. Rattus rattus. Vertebrados superiores exóticos en México: diversidad, distribución y efectos potenciales. Instituto de Ecología, Universidad Nacional Autónoma de México. Bases de datos SNIB-CONABIO. Proyecto U020. Cdad de México, México.

Amori, G., y M. Clout. 2002. Rodents on island: a conservation challenge. Pp. 6368 in Rats, mice and people: rodent, biology and management (Singleton G. R, L. A. Hinds, C. J. Krebs y D. M. Spratt, eds). Australian Centre for International Agricultural Research. Canberra, Australia.

Banks, P. B., y N. K. Hughes. 2012. A review of the evidence for potential impacts of black rats (Rattus rattus) on wildlife and humans in Australia. Wildlife Research 39:78-88.

Bertram, D., y D. Nagorsen. 1995. Introduced rats, Rattus spp. on the Queen Charlotte Islands: Implications for seabird conservation. The Canadian Field Naturalist 109:6-10.

Clark, D. B. 1980. Population ecology of Rattus rattus across a desert-montane forest gradient in the Galápagos Islands. Ecology 61:1422-1433. 
Clark, D. A. 1981. Foraging patterns of black rats across a desert-montane forest gradient in the Galapagos Islands. Biotropica 13:182-194.

Clark, D. A. 1982. Foraging behavior of a vertebrate omnivore (Rattus rattus): meal structure, sampling, and diet breadth. Ecology 63:763-772.

Dexter, N., R. C. Dowler, J. P. Flanagan, S. Hart, M. A. Revelez, y T. E. Lee. 2004. The influence of feral cats Felis catus on the distribution and abundance of introduced and endemic Galapagos rodents. Pacific Conservation Biology 10:210-215.

Feinstein, B. J. 2004. Learning and transformation in the context of Hawaian traditional ecological knowledge. Education Quarterly 54:105-20.

Gutiérrez, F. 2006. Estado de conocimiento de especies invasoras. Propuesta de lineamientos para el control de los impactos. Instituto de Investigación de Recursos Biológicos Alexander von Humboldt. Bogotá, Colombia.

Hall, E. R., y K. R. Kelson. 1959. The mammals of North America. Volume II. The Ronald Press Company. New York, EE.UU.

HaRris, D. B., y D. W. MacDonald. 2007. Interference competition between introduced black rats and endemic Galápagos rice rats. Ecology 88:2330-2344.

inefaN (Instituto Ecuatoriano Forestal y de Áreas Naturales y Vida Silvestre). 1998. Plan de Manejo Estratégico del Parque Nacional Sangay. Dirección Nacional de Áreas Naturales y Vida Silvestre. Quito, Ecuador.

iUCN (International Union for Conservation Nature). 2001. Global strategy on invasive alien species. Global Invasive Species Programme (GISP). Gland, Switzerland.

JaKsıC, F. M. 1998. Vertebrate invaders and their ecological impacts in Chile. Biodiversity and Conservation 7:1427-1445.

Lee, T. E., Jr., C. Boada-Terán, A. M. Scott, S. F. Burneo, y J. D. Hanson. 2011. Small mammals of Sangay National Park, Chimborazo Province and Morona Santiago Province, Ecuador. Occasional Papers, Museum of Texas Tech University 305:114.

Lobos, G., M. Ferres., y R. E. Palma. 2005. Presencia de los géneros invasores Mus y Rattus en áreas naturales de Chile: un riesgo ambiental y epidemiológico. Revista Chilena de Historia Natural 78: 113-124.

McChesney, G. J., y B. R. Tershy. 1998. History and status of introduced mammals and impacts to breeding seabirds on the California Channel and northwestern Baja California Islands. Colonial Waterbirds 21:335-347.

Meluink, E. 1992. The status of Neotoma anthonyi (Rodentia, Muridae, Cricetinae) of Todos Santos Islands, Baja California, Mexico. Bulletin of the Southern California Academy of Science 91:137-140.

Musser, G. G., M. D., Carleton., E. M. Brothers, y A. L. Gardner. 1998. Systematic studies of oryzomyine rodents (Muridae, Sigmodontinae): diagnoses and distributions of species formerly assigned to Oryzomys "capito". Bulletin of the American Museum of Natural History 236:1-376.

Musser, G. G., y M. D. Carleton. 2005. Superfamily Muroidea. Pp. 894-1531 in Mammal species of the World, a taxonomic and geographic reference (Wilson, D. E., y D. M. Reeder, eds.). Tercera edición. The John Hopkins University Press. Baltinore, EE.UU. 
NowAK, R. M. 1991. Walker's mammals of the world. The Johns Hopkins University Press. Baltimore, EE.UU.

OJastı, J. 2001. Especies exóticas invasoras. Estrategia regional de biodiversidad para los países del trópico andino. Convenio de Cooperación Técnica ATN/JF-5887RG CAN-BID. Caracas, Venezuela.

Pinto, C. M., M. J. Grijalva, y J. A. Costales. 2003. Prevalencia de Trypanosoma cruzi en roedores y marsupiales en dos localidades de Manabí, Ecuador. Revista de la Pontificia Universidad Católica del Ecuador 71:225-233.

Pinto, C. M., S. Ocaña-Mayorga., M. S. Lascano, y M. J Grijalva. 2006. Natural Infection of trypanosomes in marsupials and rodents associated to human dwellings in Ecuador. Journal of Parasitology 92:1251-1255.

Ruffino, L., K. Bourgeois, E. Vidal, C. Duhem, M. Paracuellos, F. Escribano, P. Sposimo, N. Baccetti, M. Pascal, y D. Oro. 2009. Invasive rats and seabirds after 2,000 years of an unwanted coexistence on Mediterranean islands. Biological Invasions 11:1631-1651.

ShePherd, J. D., y S. D. RebeCCA. 2012. Predation by Rattus norvegicus on a native small mammal in an Araucaria araucana forest of Neuquén, Argentina. Revista Chilena de Historia Natural 85:155-159.

Shiels, A. B., W. C. Pitt., R. T. Sugihara, y G. W. Witmer. 2013. Biology and Impacts of Pacific Island Invasive Species 11. The Black Rat, Rattus rattus (Rodentia: Muridae). Pacific Science 68:1-94.

TiriRA, D. 2007. Guía de campo de los mamíferos del Ecuador. Ediciones Murciélago Blanco. Publicación especial sobre los mamíferos del Ecuador 6. Quito, Ecuador.

Towsend, P., R. Scachetti-Pereira, y W. W. Hargrove. 2004. Potential geographic distribution of Anoplophora glabripennis (Coleoptera: Cerambycidae) in North America. The American Midland Naturalist 151:170-5.

Towns, D. R., I. A. E. Atkinson, y C. H. Daugherty. 2006. Have the harmful effects of introduced rats on islands been exaggerated? Biological Invasions 8:863-891.

Traveset, A., M. Nogales, J. A. Alcover, J. D. Delgado, M. Lopez-Darias, D. Godoy, J. M. IguAL, y P. Bover. 2009. A review of the effects of alien rodents in the Balearic (Western Mediterranean Sea) and Canary Islands (Eastern Atlantic Ocean). Biological Invasions 11:1653-1670.

Velarde, E., y D. W. Anderson. 1994. Conservation and management of seabird islands in the Gulf of California: Setbacks and successes. Pp 219-228 in Seabirds on Islands: threats, case studies and Action Plans (Nettleship, D .N., J. Burger y M. Gachfeld). Birdlife Conservation Series No. 1, Bird Life International. Cambridge, England.

Sometido: 17 de enero de 2014

Revisado: 19 de febrero de 2014

Aceptado: 14 de marzo de 2014

Editor asociado: Consuelo Lorenzo

Diseño gráfico editorial: Gerardo Hernández 
